\title{
Some Padé approximations and inequalities for the complete elliptic integrals of the first kind
}

\section{Mansour Mahmoud ${ }^{1 *}$ (D) and Mona Anis²}

${ }^{*}$ Correspondence:

mansour@mans.edu.eg

${ }^{1}$ Mathematics Department, Faculty

of Science, King Abdulaziz

University, P.O. Box 80203, Jeddah,

21589, Saudi Arabia

Full list of author information is

available at the end of the article

\begin{abstract}
In this paper, we present Padé approximations of some functions involving complete elliptic integrals of the first kind $K(x)$, and motivated by these approximations we also present the following double inequality:$$
\frac{1-x^{2}}{1-x^{2}+\frac{x^{4}}{62}}<\frac{2 e^{\frac{2}{\pi} K(x)-1}}{\left(1+\frac{1}{\sqrt{1-x^{2}}}\right)}<\frac{1-\frac{96}{100} x^{2}}{1-\frac{96}{100} x^{2}+\frac{x^{4}}{64}}, \quad x \in(0,1) .
$$

Our results have superiority over some new recent results.

MSC: 33E05; 26D15; 41A21

Keywords: Complete elliptic integrals; Hypergeometric function; Inequality; Padé approximant; Best possible constant; Error
\end{abstract}

\section{Springer}

\section{Introduction}

It is well known that the complete elliptic integrals of the first kind and of the second kind are classical integrals, and apart from their theoretical importance in the theory of theta functions, they have important applications in mechanics, statistical mechanics, electrodynamics, magnetic field calculations, astronomy, geodesy, quasiconformal mappings, and other fields of mathematics and mathematical physics. In most applications, we encounter complicated expressions involving the complete elliptic integrals (which are not always in a form that is immediately recognizable), and it is difficult to find numerical values of such expressions to a sufficient number of significant digits. The complete elliptic integrals cannot be expressed in terms of elementary functions and have representations as infinite series that slowly converge, so these series are not the most computationally efficient approach for most scientists and engineers. Therefore, there is a need for appropriate approximations and bounds for these integrals.

(c) The Author(s) 2021. This article is licensed under a Creative Commons Attribution 4.0 International License, which permits use, sharing, adaptation, distribution and reproduction in any medium or format, as long as you give appropriate credit to the original author(s) and the source, provide a link to the Creative Commons licence, and indicate if changes were made. The images or other third party material in this article are included in the article's Creative Commons licence, unless indicated otherwise in a credit line to the material. If material is not included in the article's Creative Commons licence and your intended use is not permitted by statutory regulation or exceeds the permitted use, you will need to obtain permission directly from the copyright holder. To view a copy of this licence, visit http://creativecommons.org/licenses/by/4.0/. 
The complete elliptic integrals of the first and second kinds $K(x)$ and $E(x)$, respectively, are defined as $[9,14]$

$$
K(x)=\int_{0}^{\frac{\pi}{2}} \frac{d t}{\sqrt{1-x^{2} \sin ^{2} t}}, \quad 0<x<1
$$

and

$$
E(x)=\int_{0}^{\frac{\pi}{2}} \sqrt{1-x^{2} \sin ^{2} t} d t, \quad 0<x<1,
$$

which satisfy

$$
\begin{aligned}
& \lim _{x \rightarrow 0^{+}} K(x)=\lim _{x \rightarrow 0^{+}} E(x)=\frac{\pi}{2}, \quad \lim _{x \rightarrow 1^{-}} K(x)=\infty, \quad \lim _{x \rightarrow 1^{-}} E(x)=1, \\
& K^{\prime}(x)=K\left(\sqrt{1-x^{2}}\right) \quad \text { and } \quad E^{\prime}(x)=E\left(\sqrt{1-x^{2}}\right) .
\end{aligned}
$$

The functions $K(x)$ and $E(x)$ have the following representation [23]:

$$
K(x)=\frac{\pi}{2} F\left(\frac{1}{2}, \frac{1}{2}, 1, x^{2}\right)
$$

and

$$
E(x)=\frac{\pi}{2} F\left(\frac{1}{2}, \frac{-1}{2}, 1, x^{2}\right)
$$

where the hypergeometric function $F(a, b, c, x)$ is defined by [5]

$$
F(a, b, c, x)=\sum_{n=0}^{\infty} \frac{(a)_{n}(b)_{n}}{(c)_{n}} \frac{x^{n}}{n !}, \quad-1<x<1,
$$

with $(a)_{n}=\frac{\Gamma(a+n)}{\Gamma(a)}$ and the Euler gamma function $\Gamma(x)$ is defined by the improper integral

$$
\Gamma(x)=\int_{0}^{\infty} e^{-v} v^{x-1} d v, \quad x>0
$$

The hypergeometric function $F(a, b, c, x)$ has the differentiation formula [5]

$$
\frac{d^{r}}{d x^{r}} F(a, b, c, x)=\frac{(a)_{r}(b)_{r}}{(c)_{r}} F(a+r, b+r, c+r, x)
$$

and the transformation

$$
(1-x)^{a+b-c} F(a, b, c, x)=F(c-a, c-b, c, x), \quad a, b, c>0 ; a+b>c .
$$

Wallis's ratio $W_{n}$ is defined as $[10,12]$

$$
W_{n}=\frac{\Gamma(n+1 / 2)}{\Gamma\left(\frac{1}{2}\right) \Gamma(n+1)}, \quad n \in \mathbb{N},
$$


and satisfies the recurrence relation

$$
W_{n+1}=\frac{n+1 / 2}{n+1} W_{n}
$$

In [31], Yang et al. show that

$$
U_{n}=\pi \sum_{k=0}^{n} \frac{W_{k}^{2} W_{n-k}^{2}}{(k+1)(n-k+1)}-\frac{6(2 n+1) W_{n}^{2}}{(n+1)(n+2)}<0, \quad n \geq 8 .
$$

$K(x)$ can be written using the notation $W_{n}$ as follows:

$$
K(x)=\frac{\pi}{2} \sum_{n=0}^{\infty} W_{n}^{2} x^{2 n}, \quad 0<x<1 .
$$

The importance of elliptic integrals led to deduction of many of their inequalities. In [11], Carlson and Gustafson presented the inequality

$$
\log \frac{4}{\sqrt{1-x^{2}}}<K(x)<\frac{4}{3+x^{2}} \log \frac{4}{\sqrt{1-x^{2}}}, \quad 0<x<1 .
$$

In [16], Kühnau deduced the lower bound

$$
K(x)>\frac{9}{8+x^{2}} \log \frac{4}{\sqrt{1-x^{2}}}, \quad 0<x<1,
$$

which is an improvement of the left-hand side of inequality (11). In [4], Anderson et al. deduced the inequality

$$
\frac{\pi}{2} \sqrt{\frac{\tanh ^{-1}(x)}{x}}<K(x)<\frac{\pi}{2} \frac{\tanh ^{-1}(x)}{x}, \quad 0<x<1 .
$$

Alzer and Qiu [1] presented the inequality

$$
\frac{\pi}{2}\left(\frac{\tanh ^{-1}(x)}{x}\right)^{\mu}<K(x)<\frac{\pi}{2}\left(\frac{\tanh ^{-1}(x)}{x}\right)^{\nu}, \quad 0<x<1,
$$

with the best possible constants $\mu=3 / 4$ and $v=1$, which improved the lower bound of (13). In [31], Yang et al. proved the inequality

$$
\log \frac{4}{\sqrt{1-x^{2}}}<K(x)<\log \left(e^{\frac{\pi}{2}}-4+\frac{4}{\sqrt{1-x^{2}}}\right), \quad 0<x<1 .
$$

In 2019, Yang and Tian [32] deduced the inequality

$$
\rho \log \left(1+\frac{4}{\sqrt{1-x^{2}}}\right)<K(x)<\sigma \log \left(1+\frac{4}{\sqrt{1-x^{2}}}\right),
$$

with the best possible constants $\rho=\frac{\pi}{2 \ln 5}$ and $\sigma=1$. Recently, Wang et al. [27] presented the inequality

$$
K(x)<\log \left(1+\frac{4}{\sqrt{1-x^{2}}}\right)\left[\frac{\pi}{2 \log 5}+\left(1-\frac{\pi}{2 \log 5}\right) x^{2}\right], \quad 0<x<1 .
$$


For more details about inequalities, applications, and other related special functions to $K(x)$ and $E(x)$, we refer to $[2,3,13,15,17-22,24-26,28-30]$ and the references therein.

Padé approximant [6-8] of order $(r, s)$ of a function $f(x)$ is a rational function

$$
[r / s]_{f}(x)=\frac{\sum_{i=0}^{r} \alpha_{i} x^{i}}{1+\sum_{i=1}^{s} \beta_{i} x^{i}}, \quad r ; s \geq 0
$$

where singularities of $f(x)$ are only poles. There are many different ways to determine the other coefficients $\alpha_{j} s$ for $0 \leq j \leq r$ and $\beta_{k} s$ for $1 \leq k \leq s$. Among them is the matching between the first $r+s+1$ coefficients in Maclaurin series $f(x)=\sum_{k=0}^{\infty} c_{k} x^{k}$ and the first $r+s+1$ coefficients of Padé approximant by the relation

$$
\sum_{k=0}^{r+s+1} c_{k} x^{k}=\frac{\sum_{i=0}^{r} \alpha_{i} x^{i}}{\sum_{i=0}^{s} \beta_{i} x^{i}} \quad \text { or } \quad\left(\sum_{k=0}^{r+s+1} c_{k} x^{k}\right)\left(\sum_{i=0}^{s} \beta_{i} x^{i}\right)=\left(\sum_{i=0}^{r} \alpha_{i} x^{i}\right) .
$$

Hence, we solve the following equations for $\alpha_{i} s$ and $\beta_{i} s$ :

$$
c_{r+1}+\sum_{k=1}^{s} c_{k} \beta_{r+1-k}=0 \quad \text { and } \quad \alpha_{r}=\sum_{k=0}^{r} \beta_{r-k} c_{k}
$$

and we have

$$
[r / s]_{f}(x)-f(x)=O\left(x^{r+s+1}\right)
$$

\section{Main results}

Theorem 1 The following inequality

$$
K(x)<\frac{\pi}{2} \log \left[\frac{e}{p+1}\left(p+\frac{1}{\sqrt{1-x^{2}}}\right)\right], \quad 0<x<1
$$

holds for the best possible constant $p=1$.

Proof Consider the function

$$
F_{p}(x)=\frac{e^{\frac{2 K(x)}{\pi}}}{p+\frac{1}{\sqrt{1-x^{2}}}} .
$$

Using (4) and (6), we have

$$
F_{p}^{\prime}(\sqrt{x})=\frac{e^{F\left(\frac{1}{2}, \frac{1}{2}, 1, x\right)}}{4\left(p+\frac{1}{\sqrt{1-x}}\right)^{2}}\left[\left(p+\frac{1}{\sqrt{1-x}}\right) F\left(\frac{3}{2}, \frac{3}{2}, 2, x\right)-\frac{2}{(1-x)^{\frac{3}{2}}}\right]
$$

and then the function $F_{p}(\sqrt{x})$ is strictly decreasing on $x \in(0,1)$ if and only if

$$
p \leq \frac{2}{F\left(\frac{3}{2}, \frac{3}{2}, 2, x\right)(1-x)^{\frac{3}{2}}}-\frac{1}{\sqrt{1-x}} \doteqdot f(x) .
$$


Using relation (7), we have

$$
f(x)=\frac{2-F\left(\frac{1}{2}, \frac{1}{2}, 2, x\right)}{\sqrt{1-x} F\left(\frac{1}{2}, \frac{1}{2}, 2, x\right)},
$$

and hence

$$
f^{\prime}(x)=\frac{1}{4 F\left(\frac{1}{2}, \frac{1}{2}, 2, x\right)^{2}(1-x)^{\frac{3}{2}}} f_{1}(x),
$$

where

$$
f_{1}(x)=4 F\left(\frac{1}{2}, \frac{1}{2}, 2, x\right)-2 F\left(\frac{1}{2}, \frac{1}{2}, 2, x\right)^{2}-(1-x) F\left(\frac{3}{2}, \frac{3}{2}, 3, x\right) .
$$

From (8), we have

$$
\begin{aligned}
f_{1}(x) & =4 \sum_{n=0}^{\infty} \frac{W_{n}^{2}}{n+1} x^{n}-2\left(\sum_{n=0}^{\infty} \frac{W_{n}^{2}}{n+1} x^{n}\right)^{2}+\sum_{n=0}^{\infty} \frac{2(4 n-1) W_{n}^{2}}{(n+1)(n+2)} x^{n} \\
& =-2 \sum_{n=0}^{\infty} \sum_{k=0}^{n} \frac{W_{k}^{2} W_{n-k}^{2}}{(k+1)(n-k+1)} x^{n}+\sum_{n=0}^{\infty} \frac{6(2 n+1) W_{n}^{2}}{(n+1)(n+2)} x^{n} \\
& =-\sum_{n=0}^{\infty} \mu_{n} x^{n},
\end{aligned}
$$

where

$$
\mu_{n}=2 \sum_{k=0}^{n} \frac{W_{k}^{2} W_{n-k}^{2}}{(k+1)(n-k+1)}-\frac{6(2 n+1) W_{n}^{2}}{(n+1)(n+2)} .
$$

Using (9), we obtain

$$
\mu_{n}<U_{n}<0, \quad n \geq 8
$$

and $\mu_{0}=-1, \mu_{1}=\frac{-1}{4}, \mu_{2}=\frac{-17}{128}, \mu_{3}=\frac{-43}{512}, \mu_{4}=\frac{-953}{16,384}, \mu_{5}=\frac{-2801}{65,536}, \mu_{6}=\frac{-137,401}{4,194,304}, \mu_{7}=$ $\frac{-485,318}{16,777,216}$. Hence $\mu_{n}<0$ for $n \geq 0, f_{1}(x)>0$ and therefore the function $f(x)$ is increasing on $x \in(0,1)$ with

$$
\lim _{x \rightarrow 0^{+}} f(x)=1,
$$

which implies that $p \leq 1$. Therefore, the function $F_{p}(x)$ is strictly decreasing on $x \in(0,1)$ if and only if $p \leq 1$, and using the first limit in (3), we obtain inequality (18).

Theorem 2 The following inequality

$$
K(x)>\frac{\pi}{2} \log \left[\frac{e}{q+1}\left(q+\frac{1}{\sqrt{1-\frac{11}{12} x^{2}}}\right)\right], \quad 0<x<1
$$

holds for the best possible constant $q=\frac{5}{6}$. 
Proof Consider the function

$$
H_{q}(x)=\frac{e^{\frac{2 K(x)}{\pi}}}{q+\frac{1}{\sqrt{1-\frac{11}{12} x^{2}}}}
$$

Using (4) and (6), we have

$$
H_{q}^{\prime}(x)=\frac{e^{F\left(\frac{1}{2}, \frac{1}{2}, 1, x\right)}}{4\left(q+\frac{1}{\sqrt{1-\frac{11}{12} x}}\right)^{2}}\left[\left(q+\frac{1}{\sqrt{1-\frac{11}{12} x}}\right) F\left(\frac{3}{2}, \frac{3}{2}, 2, x\right)-\frac{11}{6\left(1-\frac{11}{12} x\right)^{\frac{3}{2}}}\right],
$$

and then the function $H_{q}(\sqrt{x})$ is strictly increasing on $(0,1)$ if and only if

$$
q \geq \frac{11}{6 F\left(\frac{3}{2}, \frac{3}{2}, 2, x\right)\left(1-\frac{11}{12} x\right)^{\frac{3}{2}}}-\frac{1}{\sqrt{1-\frac{11}{12} x}} \doteqdot h(x)
$$

Then

$$
h^{\prime}(x)=\frac{11 \sqrt{3}}{2 F\left(\frac{3}{2}, \frac{3}{2}, 2, x\right)^{2}(12-11 x)^{\frac{5}{2}}} h_{1}(x),
$$

where

$$
h_{1}(x)=132 F\left(\frac{3}{2}, \frac{3}{2}, 2, x\right)+(-24+22 x) F\left(\frac{3}{2}, \frac{3}{2}, 2, x\right)^{2}+9(-12+11 x) F\left(\frac{5}{2}, \frac{5}{2}, 3, x\right) \text {. }
$$

From (8), we have

$$
\begin{aligned}
h_{1}(x)= & 132 \sum_{n=0}^{\infty} \frac{(2 n+1)^{2} W_{n}^{2}}{n+1} x^{n}+(-24+22 x)\left(\sum_{n=0}^{\infty} \frac{(2 n+1)^{2} W_{n}^{2}}{n+1} x^{n}\right)^{2} \\
& +9(-12+11 x) \sum_{n=0}^{\infty} \frac{2(2 n+1)^{2}(2 n+3)^{2} W_{n}^{2}}{9(n+1)(n+2)} x^{n} \\
= & 4 \sum_{n=1}^{\infty} \sum_{k=0}^{n} \frac{-2(2 k+1)^{2}\left(\left(n-k+\frac{1}{2}\right)^{2}+\frac{11}{4}\right) W_{k}^{2} W_{n-k}^{2}}{(k+1)(n-k+1)} x^{n} \\
& -4 \sum_{n=1}^{\infty} \frac{(2 n+1)^{2}\left(2 n^{2}-5 n-12\right) W_{n}^{2}}{(n+1)(n+2)} x^{n} \\
= & 4 \sum_{n=1}^{\infty} V_{n} x^{n}
\end{aligned}
$$

and

$$
V_{n}=\sum_{k=0}^{n} \frac{-2(2 k+1)^{2}\left(\left(n-k+\frac{1}{2}\right)^{2}+\frac{11}{4}\right) W_{k}^{2} W_{n-k}^{2}}{(k+1)(n-k+1)}-\frac{(2 n+1)^{2}(2 n+3)(n-4) W_{n}^{2}}{(n+1)(n+2)}
$$

The sequence $V_{n}<0$ for $n=4,5,6, \ldots$ and

$$
V_{0}=0, \quad V_{1}=\frac{-19}{8}, \quad V_{2}=\frac{-663}{128}, \quad V_{3}=\frac{-8367}{1024}
$$


Then $V_{n}<0$ for $n \geq 0, h_{1}(x)<0$ and therefore the function $h(x)$ is decreasing with

$$
\lim _{x \rightarrow 0^{+}} h(x)=\frac{5}{6}
$$

which implies that $q \geq \frac{5}{6}$. Therefore, the function $H_{q}(x)$ is strictly increasing on $x \in(0,1)$ if and only if $q \geq \frac{5}{6}$, and using the limits in (3), we obtain inequality (19).

Based on the Padé approximation method, we can conclude the following approximations.

Proposition 3 The Padé approximations of orders $(3,4)$ and $(3,7)$ of the function

$$
\begin{aligned}
f(x) & =\frac{2 e^{\frac{2}{\pi} K(x)-1}}{\left(1+\frac{1}{\sqrt{1-x^{2}}}\right)} \\
& =1-\frac{x^{4}}{64}-\frac{13 x^{6}}{768}-\frac{261 x^{8}}{16,384}-\frac{14,317 x^{10}}{983,040}+\cdots, \quad x \rightarrow 0
\end{aligned}
$$

are the following rational functions:

$$
[3 / 4]_{f}(x)=\frac{1-\frac{13 x^{2}}{12}}{1-\frac{13 x^{2}}{12}+\frac{x^{4}}{64}}+O\left(x^{8}\right)
$$

and

$$
[3 / 7]_{f}(x)=\frac{1-\frac{795 x^{2}}{832}}{1-\frac{795 x^{2}}{832}+\frac{x^{4}}{64}+\frac{319 x^{6}}{159,744}}+O\left(x^{11}\right) .
$$

Proposition 4 The Padé approximations of orders $(3,7)$ and $(3,9)$ of the function

$$
\begin{aligned}
g(x) & =\frac{11 e^{\frac{2}{\pi} K(x)-1}}{12\left(\frac{1}{\frac{5}{6}+\sqrt{1-\frac{11 x^{2}}{12}}}\right)} \\
& =\frac{1}{2}+\frac{19 x^{6}}{9216}+\frac{403 x^{8}}{110,592}+\frac{167,659 x^{10}}{35,389,440}+\frac{1,862,857 x^{12}}{339,738,624}+\cdots, \quad x \rightarrow 0
\end{aligned}
$$

are the following rational functions:

$$
[3 / 7]_{g}(x)=\frac{\frac{1}{2}-\frac{403 x^{2}}{456}}{-\frac{19 x^{6}}{4608}-\frac{403 x^{2}}{228}+1}+O\left(x^{11}\right)
$$

and

$$
[3 / 9]_{g}(x)=\frac{\frac{1}{2}-\frac{167,659 x^{2}}{257,920}}{1-\frac{167,659 x^{2}}{128,960}-\frac{19 x^{6}}{4608}-\frac{3,436,157 x^{8}}{1,782,743,040}}+O\left(x^{13}\right) .
$$

Unfortunately, formulas (20),(21), (22), and (23) did not give bounds of the function $K(x)$ for all $x$ in the domain $(0,1)$. But formula (20) motivates us to establish the following inequalities. 
Theorem 5 The following inequality

$$
K(x)<\frac{\pi}{2} \log \left(\frac{e}{2}\left(1+\frac{1}{\sqrt{1-x^{2}}}\right)\left(\frac{1-\frac{96}{100} x^{2}}{1-\frac{96}{100} x^{2}+\frac{x^{4}}{64}}\right)\right)
$$

holds for $0<x<1$.

Proof Consider the function

$$
T(x)=\frac{e^{\frac{2 K(x)}{\pi}}}{\frac{e}{2}\left(1+\frac{1}{\sqrt{1-x^{2}}}\right)\left(\frac{1-\frac{96}{100} x^{2}}{1-\frac{96}{100} x^{2}+\frac{x^{4}}{64}}\right)},
$$

and hence

$$
T^{\prime}(\sqrt{x})=\frac{(-1600+(1536-25 x) x) e^{F\left(\frac{1}{2}, \frac{1}{2}, 1, x\right)}}{128 e \sqrt{1-x}(-25+24 x)(1+\sqrt{1-x})} \frac{t_{1}(x)}{\left(x^{2}-1\right)},
$$

where

$$
t_{1}(x)=w_{1}(x)-w_{2}(x)
$$

with

$$
w_{1}(x)=\left(x^{2}-1\right) F\left(\frac{1}{2}, \frac{1}{2}, 2, x\right)
$$

and

$$
\begin{aligned}
w_{2}(x)= & \frac{\left(x^{2}-1\right)}{(1+\sqrt{1-x})(25-24 x)(1600-(1536-25 x) x)}[80,000 \\
& -200(793+25 \sqrt{1-x}) x+(82,378+7400 \sqrt{1-x}) x^{2} \\
& \left.-1200(3+2 \sqrt{1-x}) x^{3}\right] .
\end{aligned}
$$

Now

$$
\begin{aligned}
w_{1}(x) & =\sum_{n=0}^{\infty} \frac{W_{n}^{2}}{n+1} x^{n+2}-\sum_{n=0}^{\infty} \frac{W_{n}^{2}}{n+1} x^{n} \\
& =-1-\frac{x}{8}+\sum_{n=2}^{\infty} \frac{\left(64 n^{3}-104 n^{2}+48 n-9\right) W_{n}^{2}}{(n+1)(2 n-1)^{2}(2 n-3)^{2}} x^{n}
\end{aligned}
$$

and

$$
w_{1}^{\prime \prime}(x)=\sum_{n=0}^{\infty} \frac{(n+1)(n+2)\left(183+400 n+280 n^{2}+64 n^{3}\right) W_{n+2}^{2}}{(n+3)(3+4 n(n+2))^{2}} x^{n} .
$$

Then $w_{1}(x)$ is a convex function between the points $(0,-1)$ and $(1,0)$. Also,

$$
w_{2}^{\prime \prime}\left(1-u^{2}\right)=\frac{w_{3}(u)}{w_{4}(u)}, \quad 0<u<1,
$$


where

$$
w_{4}(u)=-2 u\left(89+3622 u^{2}+35,689 u^{4}+600 u^{6}\right)^{3}(1+u)^{3}<0
$$

and

$$
\begin{aligned}
w_{3}(u)= & -422,9814-171,806,490 u-1,017,032,637 u^{2}-107,1925,167 u^{3} \\
& -26,188,833,484 u^{4}+167,770,858,692 u^{5}-106,178,812,935 u^{6} \\
& +2,315,906,378,995 u^{7}-2,725,881,138,990 u^{8}-8,235,729,120,210 u^{9} \\
& -93,146,282,277,931 u^{10}-135,010,050,058,665 u^{11}-381,354,896,855,688 u^{12} \\
& -469,576,678,642,848 u^{13}-381,450,253,571,121 u^{14} \\
& -157,869,853,296,507 u^{15}-117,933,228,362,600 u^{16} \\
& -118,127,740,267,800 u^{17}-38,943,594,964,800 u^{18}-1,971,366,120,000 u^{19} \\
& -629,017,920,000 u^{20}-11,016,000,000 u^{21}-3,456,000,000 u^{22} \\
= & (1-u)^{24} \int_{0}^{\infty} e^{(1-u) t} w_{5}(t) d t<0,
\end{aligned}
$$

where

$$
\begin{aligned}
w_{5}(t)= & -\frac{1,937,500 t^{23}}{26,298,031,350,591}-\frac{415,555,625 t^{22}}{18,294,282,678,672}-\frac{154,777,075 t^{21}}{48,915,194,328} \\
& -\frac{167,679,321,125 t^{20}}{633,568,231,296}-\frac{90,786,961,225 t^{19}}{6,092,002,224}-\frac{100,101,056,444,021 t^{18}}{166,728,481,920} \\
& -\frac{3,562,788,612,574,819 t^{17}}{198,486,288,000}-\frac{133,198,651,249,637,299 t^{16}}{326,918,592,000} \\
& -\frac{2,692,769,780,390,699 t^{15}}{378,378,000}-\frac{174,766,864,343,435,699 t^{14}}{1,816,214,400} \\
& -\frac{43,522,631,386,179,371 t^{13}}{43,243,200}-\frac{5,123,113,557,369,119 t^{12}}{633,600} \\
& -\frac{122,852,110,141,292,563 t^{11}}{2,494,800}-\frac{809,450,870,289,838,177 t^{10}}{3,628,800} \\
& -\frac{2,111,790,435,345,101 t^{9}}{2880}-\frac{68,468,628,875,595,707 t^{8}}{40,320} \\
& -\frac{169,054,344,340,120 t^{7}}{63}-\frac{5,639,548,747,095 t^{6}}{2}-1,965,373,967,040 t^{5} \\
& -924,471,855,000 t^{4}-276,448,320,000 t^{3}-43,524,000,000 t^{2} \\
& 3,456,000,000 t<0 .
\end{aligned}
$$

Then $w_{2}(x)$ is a convex function between the same two points $(0,-1)$ and $(1,0)$. Also,

$$
\lim _{x \rightarrow 0^{+}} w_{2}^{\prime}(x)=\frac{-1}{8}, \quad \lim _{x \rightarrow 0^{+}} w_{1}^{\prime}(x)=\frac{-1}{8}
$$


and

$$
\lim _{x \rightarrow 1^{-}} w_{2}^{\prime}(x)=4, \quad \lim _{x \rightarrow 1^{-}} w_{1}^{\prime}(x)=\frac{8}{\pi} .
$$

Then $w_{1}(x)>w_{2}(x), t_{1}(x)>0$ and $T(x)$ is decreasing on $x \in(0,1)$. Hence, using the limits in (3), we obtain inequality (24).

Theorem 6 The inequality

$$
K(x)>\frac{\pi}{2} \log \left(\frac{e}{2}\left(1+\frac{1}{\sqrt{1-x^{2}}}\right)\left(\frac{1-x^{2}}{1-x^{2}+\frac{x^{4}}{62}}\right)\right)
$$

holds for $x \in(0,1)$.

Proof Consider the function

$$
G(x)=\frac{e^{\frac{2 K(x)}{\pi}}}{\frac{e}{2}\left(1+\frac{1}{\sqrt{1-x^{2}}}\right)\left(\frac{1-x^{2}}{1-x^{2}+\frac{x^{4}}{62}}\right)}
$$

and hence

$$
G^{\prime}(\sqrt{x})=\frac{\left(62-62 x+x^{2}\right) e^{F\left(\frac{1}{2}, \frac{1}{2}, 1, x\right)}}{124 e(1-x)^{\frac{3}{2}}(1+\sqrt{1-x})} g_{1}(x),
$$

where

$$
g_{1}(x)=F\left(\frac{1}{2}, \frac{1}{2}, 2, x\right)-\frac{2\left(62-2(33+2 \sqrt{1-x}) x+(3+2 \sqrt{1-x}) x^{2}\right.}{(1+\sqrt{1-x})\left(62-62 x+x^{2}\right)} .
$$

Using (5), we get

$$
F\left(\frac{1}{2}, \frac{1}{2}, 2, x\right)=\sum_{n=0}^{\infty} \frac{W_{n}^{2}}{n+1} x^{n}>1+\frac{x}{8}+\frac{3 x^{2}}{64}+\frac{25 x^{3}}{1024} .
$$

Now let

$$
\begin{gathered}
g_{2}(x)=(1+\sqrt{1-x})^{2}\left(2\left(62-2(33+2 \sqrt{1-x}) x+(3+2 \sqrt{1-x}) x^{2}\right)\right. \\
\left.-\left(1+\frac{x}{8}+\frac{3 x^{2}}{64}+\frac{25 x^{3}}{1024}\right)(1+\sqrt{1-x})\left(62-62 x+x^{2}\right)\right) . \\
g_{2}^{\prime}\left(1-u^{2}\right)=\frac{g_{3}(u)}{2048 u}, \quad 0<u<1,
\end{gathered}
$$

where

$$
\begin{aligned}
g_{3}(u)= & -1225-114,096 u+533,640 u^{2}-875,268 u^{3}+489,925 u^{4}+154,176 u^{5} \\
& -194,208 u^{6}-67,464 u^{7}+75,897 u^{8}+13,520 u^{9}-14,872 u^{10}+300 u^{11}
\end{aligned}
$$




$$
\begin{aligned}
& -325 u^{12}-194,208 u^{6}-67,464 u^{7}+75,897 u^{8}+13,520 u^{9}-14,872 u^{10} \\
& +300 u^{11}-325 u^{12} \\
= & (1-u)^{14} \int_{0}^{\infty} e^{(1-u) t} g_{4}(t) d t \\
< & 0
\end{aligned}
$$

where

$$
\begin{aligned}
g_{4}(t)= & -\frac{1}{623,700}\left[48 t^{11}+6864 t^{10}+313,225 t^{9}+5,268,780 t^{8}\right. \\
& +85,446,900 t^{7}+794,011,680 t^{6}+3,030,340,005 t^{5}+4,942,822,500 t^{4} \\
& \left.+3,432,636,900 t^{3}+1,122,660,000 t^{2}+202,702,500 t\right] .
\end{aligned}
$$

Then $g_{2}(x)$ is deceasing with

$$
\lim _{x \rightarrow 0^{+}} g_{2}(x)=0 .
$$

Hence $g_{2}(x)<0$, and we have

$$
\frac{2\left(62-2(33+2 \sqrt{1-x}) x+(3+2 \sqrt{1-x}) x^{2}\right.}{(1+\sqrt{1-x})\left(62-62 x+x^{2}\right)}<1+\frac{x}{8}+\frac{3 x^{2}}{64}+\frac{25 x^{3}}{1024} .
$$

From inequalities (26) and (27), we get $g_{1}(x)>0$ and the function $G(x)$ is increasing. Hence, using the limits in (3), we obtain inequality (25)

\section{Remarks}

Comparing our new bounds of the function $K(x)$ with its previous ones presents the following remarks.

Remark 7 Our upper bound in (24) is better than our upper bound in (18) for $x \in(0,1)$.

Remark 8 The upper bound in (24) is better than the upper bound in (11) for $x \in(0,0.97)$.

Remark 9 The upper bound in (24) is better than the upper bound in (14) for $x \in(0,1)$.

Remark 10 The upper bound in (24) is better than the upper bound in each of (15), (16), and (17) for $x \in(0,0.98)$.

Remark11 Our lower bounds in (19) and (25) are not contained in each other for $x \in(0,1)$.

Remark 12 Our lower bound in (25) is better than the lower bound in (12) for $x \in(0,9)$.

Remark 13 The lower bound in (25) is better than the lower bound in (14) for $x \in(0,87)$.

Remark 14 The lower bound in (25) is better than the lower bound in (15) for $x \in(0,94)$.

Remark 15 The lower bound in (25) is better than the lower bound in (16) for $x \in(0,91)$. 
Acknowledgements

Not applicable.

\section{Funding}

Not applicable.

\section{Availability of data and materials}

The data used to support the findings of this study are available from the corresponding author upon request.

\section{Competing interests}

The authors declare that they have no competing interests.

\section{Authors' contributions}

All authors contributed equally and significantly in writing this article. All authors read and approved the final manuscript.

\section{Author details}

${ }^{1}$ Mathematics Department, Faculty of Science, King Abdulaziz University, P.O. Box 80203, Jeddah, 21589, Saudi Arabia.

${ }^{2}$ Mathematics Department, Faculty of Science, Mansoura University, Mansoura, 35516, Egypt.

\section{Publisher's Note}

Springer Nature remains neutral with regard to jurisdictional claims in published maps and institutional affiliations.

\section{Received: 29 August 2020 Accepted: 9 February 2021 Published online: 19 February 2021}

\section{References}

1. Alzer, H., Qiu, S.L.: Monotonicity theorems and inequalities for the complete elliptic integrals. J. Comput. Appl. Math. $172,289-312(2004)$

2. Alzer, H., Richards, K.: Inequalities for the ratio of complete elliptic integrals. Proc. Am. Math. Soc. 145(4), 1661-1670 (2017)

3. Anderson, G.D., Qiu, S.-L., Vamanamurthy, M.K.: Elliptic integral inequalities, with applications. Constr. Approx. 14(2), 195-207 (1998)

4. Anderson, G.D., Vamanamurthy, M.K., Vuorinen, M.: Functional inequalities for hypergeometric functions and complete elliptic integrals. SIAM J. Math. Anal. 23(2), 512-524 (1992)

5. Andrews, G.E., Askey, R.A., Roy, R.: Special Functions. Encyclopedia of Mathematics and Its Applications, vol. 71. Cambridge University Press, Cambridge (1999)

6. Baker, G.A. Jr., Graves-Morris, P.: Padé Approximants, 2nd edn. Cambridge University Press, Cambridge (1996)

7. Brezinski, C.: Rational approximation to formal power series. J. Approx. Theory 25, 295-317 (1979)

8. Brezinski, C.: Padé-Type Approximation and General Orthogonal Polynomials. ISNM, vol. 50. Birkhäuser, Basel (1980)

9. Byrd, P.F., Friedman, M.D.: Handbook of Elliptic Integrals for Engineers and Scientists. Springer, New York (1971)

10. Cao, X., Wang, R.: Some inequalities for the ratio of gamma functions. Arch. Inequal. Appl. 2015, 178 (2015)

11. Carlson, B.C., Gustafson, J.L.: Asymptotic expansion of the first elliptic integral. SIAM J. Math. Anal. 16(5), 1072-1092 (1985)

12. Chen, C.P., Qi, F.: Completely monotonic function associated with the gamma functions and proof of Wallis inequality. Tamkang J. Math. 36(4), 303-307 (2005)

13. Chu, Y.-M., Wang, M.-K., Qiu, S.-L.: Optimal combinations bounds of root-square and arithmetic means for Toader mean. Proc. Indian Acad. Sci. Math. Sci. 122(1), 41-51 (2012)

14. Hancock, H.: Elliptic Integrals. Dover, New York (1958)

15. Huang, T.-R., Tan, S.-Y., Ma, X.-Y., Chu, Y.-M.: Monotonicity properties and bounds for the complete $p$-elliptic integrals. J. Inequal. Appl. 2018, 239 (2018)

16. Kühnau, R.: Eine methode die Positivität einer Funktion zu prüfen. Z. Angew. Math. Mech. 74, 140-143 (1994)

17. Li, Y.-M., Xia, W.-F., Chu, Y.-M., Zhang, X.-H.: Optimal lower and upper bounds for the geometric convex combination of the error function. J. Inequal. Appl. 2015, 382 (2015)

18. Milovanović, G.V., Rassias, M.T. (eds.): Analytic Number Theory, Approximation Theory and Special Functions Springer, Berlin (2014)

19. Mitrinovic, D.S., Pecaric, J.E., Fink, A.M.: Inequalities Involving Functions and Their Integrals and Derivatives. Kluwer Academic, Dordrecht (1991)

20. Mitrinovic, D.S., Pecaric, J.E., Fink, A.M.: Classical and New Inequalities in Analysis. Kluwer Academic, Dordrecht (1993)

21. Qi, F., Huang, Z.: Inequalities of the complete elliptic integrals. Tamkang J. Math. 29(3), 165-169 (1998)

22. Qian, W.-M., He, Z.-Y., Chu, Y.-M.: Approximation for the complete elliptic integral of the first kind. Rev. R. Acad. Cienc. Exactas Fís. Nat., Ser. A Mat. 114(2), Article ID 57 (2020)

23. Rainville, E.: Special Functions. Macmillan Co., New York (1960)

24. Song, Y.-Q., Khan, M.A., Ullah, S.Z., Chu, Y.-M.: Integral inequalities involving strongly convex functions. J. Funct. Spaces 2018, Aritcle ID $6595921(2018)$

25. Vamanamurthy, M.K., Vuorinen, M.: Inequalities for means. J. Math. Anal. Appl. 183(1), 155-166 (1994)

26. Wang, M.-K., Chu, H.-H., Chu, Y.-M.: Precise bounds for the weighted Hölder mean of the complete $p$-elliptic integrals. J. Math. Anal. Appl. 480(2), Article ID 123388 (2019)

27. Wang, M.K., Chu, H.H., Li, Y.M., Chu, Y.M.: Answers to three conjectures on convexity of three functions involving complete elliptic integrals of the first kind. Appl. Anal. Discrete Math. 14, 255-271 (2020) https://doi.org/10.2298/AADM190924020W

28. Wang, B., Luo, C.-L., Li, S.-H., Chu, Y.-M.: Sharp one-parameter geometric and quadratic means bounds for the Sándor-Yang means. Rev. R. Acad. Cienc. Exactas Fís. Nat., Ser. A Mat. 114(1), Article ID 7 (2020) 
29. Wang, H., Qian, W.-M., Chu, Y.-M.: Optimal bounds for Gaussian arithmetic geometric mean with applications to complete elliptic integral. J. Funct. Spaces 2016, Article ID 3698463 (2016)

30. Wang, M.-K., Zhang, W., Chu, Y.-M.: Monotonicity, convexity and inequalities involving the generalized elliptic integrals. Acta Math. Sci. 39B(5), 1440-1450 (2019)

31. Yang, Z.H., Mao, W.M., Chu, Y.M.: Monotonicity properties and bounds involving the complete elliptic integral of first kind. Math. Inequal. Appl. 21(4), 1185-1199 (2018)

32. Yang, Z.H., Tian, J.F.: Convexity and monotonicity for elliptic integrals of the first kind and applications. Appl. Anal. Discrete Math. 13, 240-260 (2019)

Submit your manuscript to a SpringerOpen ${ }^{\circ}$ journal and benefit from:

- Convenient online submission

- Rigorous peer review

- Open access: articles freely available online

- High visibility within the field

- Retaining the copyright to your article

Submit your next manuscript at $\gg$ springeropen.com 